\title{
A global map of gene function
}

Researchers map functional interactions for $75 \%$ of the yeast genome using synthetic genetic array methodology.

Proteins carry out their functions by interacting with each other. But proteins associated with a pathway or biological process do not necessarily interact physically, so protein-protein interaction studies are restricted in regard to fully mapping gene function. By looking at functional interactions at the genetic level, researchers can learn a lot about what genes do and potentially can understand how genotype translates to phenotype.

Brenda Andrews and Charles

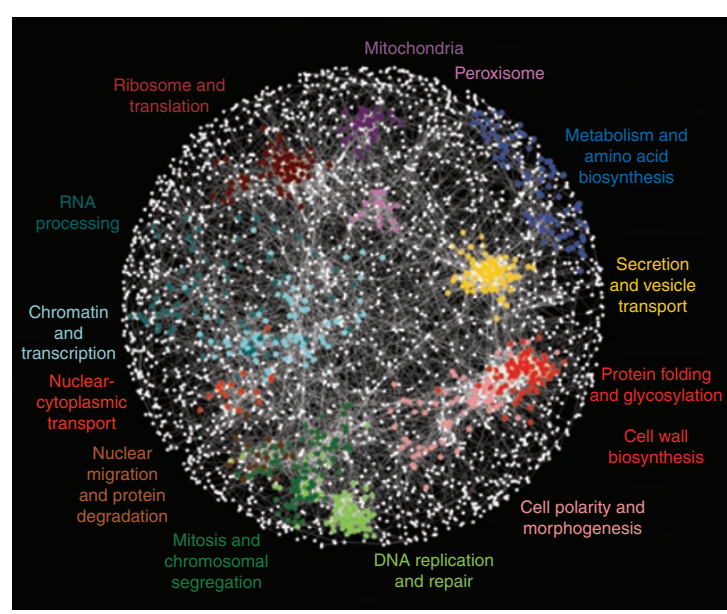

A yeast genetic interaction map. Color-coded genes with similar genetic interaction patterns are in close proximity; less similar genes are further apart. Reprinted with permission from the American Association for the Advancement of Science.
Boone of the University of Toronto, with Chad Myers of the University of Minnesota, have been using such an approach for some time. Applying their synthetic genetic array (SGA) methodology first reported in 2001, they now report a large-scale genetic interaction map for the budding yeast Saccharomyces cerevisiae.

The SGA methodology is a high-throughput approach to systematically test the effects of double mutations on the fitness of the yeast, as monitored by colony growth. The majority of genes in yeast are nonessential: that is, if these genes are mutated, other genes will cover for them and the yeast will survive. The SGA method works by identifying pairs of genes for which mutation of both genes negatively affects the fitness of the yeast, indicating that the two genes are likely to be involved in the same biological process.

In their most recent work, Andrews, Boone and their colleagues developed a scoring method for yeast fitness based on colony size. They queried 1,712 genes, about $30 \%$ of the yeast genome, by crossing them with a deletion strain collection, which generated at least partial genetic interaction profiles for $75 \%$ of the genome. From this information they generated a large-scale functional network map, which provides a powerful way to predict gene function. "You can take this global map and overlay all of the uncharacterized genes and look at where they fall on the network," explains Andrews.

The researchers took advantage of existing large-scale datasets of yeast protein- protein interactions to make comparisons. Although there were overlapping interactions between the methodologies, only a relatively small fraction of gene pairs that showed a functional interaction were actually found to physically interact, demonstrating the need for such complementary approaches.

They also found a significant correlation between genetic interactions and chemicalgenetic interactions (gene deletions that result in hypersensitivity to chemical compounds), indicating that the same genes are involved in protecting the cell against both genetic and environmental assaults. Moreover, for a given compound, the overlap of chemical-genetic and genetic interactions often predicts the "target pathway and possibly the target itself," explains Boone.

Andrews and Boone hope that researchers outside the yeast community will explore their data. "I think we might learn a lot about genetic interactions just from many people thinking about these data in different ways," says Andrews. They also emphasize that the general approach could be adapted to study different types of genetic interactions, phenotypes and organisms. Says Boone, "The goal is definitely to do it in higher-order cells, like mammalian cells."

\section{Allison Doerr}

\section{RESEARCH PAPERS}

Costanzo, M. et al. The genetic landscape of a cell. Science 327, 425-431 (2010). 